baseline, clinical data including alcohol and smoking history, BMI and features of portal hypertension were recorded. Laboratory data (bilirubin, creatinine, sodium, PT, INR and platelet count) were collected. On biopsy, the presence of cirrhosis and histological features (fat severity, lymphocyte and neutrophil infiltration) were scored semi-quantitatively.

Results Median age was 51 (29-67) and the majority (72\%) were male. All patients had a history of alcohol excess ( $>80 \mathrm{~g} /$ day for men, $50 \mathrm{~g} / \mathrm{d}$ for women). Patients were followed until death $(\mathrm{n}=97$; median $62 \mathrm{~m})$, OLT $(n=5$; median $96 \mathrm{~m})$ or are still alive $(n=32$; median $187 \mathrm{~m}$ ). Overall, the 5, 10 and 15-year survival was 64, 40 and $26 \%$, respectively. Patient baseline characteristics are shown according to outcome (Abstract PWE-285 table 1). In multivariate analysis age $(p=0.01)$, smoking $(p=0.01)$, persistent drinking $(p<0.01)$ and serum albumin at baseline $(p=0.02)$ were associated with significantly increased risk of death. No histological features correlated with prognosis.

Abstract PWE-285 Table 1 Characteristics of cohort according to 15 -year survival

\begin{tabular}{lclll}
\hline Characteristic & Alive $\mathbf{n}=\mathbf{3 2}$ & Dead $\mathbf{n}=\mathbf{1 0 2}$ & $\mathbf{0 R}$ (95\% CI) & p Value \\
\hline Age $^{*}$ & $48(44-54)$ & $53(47-58)$ & $1.08(1.02$ to 1.13$)$ & 0.01 \\
Male gender & $18(56.3)$ & $78(76.5)$ & $2.53(1.10$ to 5.83$)$ & 0.03 \\
Smoker & $12(50.0)$ & $72(72.7)$ & $2.67(1.07$ to 6.65$)$ & 0.04 \\
Subsequent abstinence & $13(41.9)$ & $17(16.8)$ & $0.28(0.12$ to 0.687$)$ & 0.01 \\
Ascites & $2(7.1)$ & $20(20.8)$ & $3.42(0.75$ to 15.64$)$ & 0.11 \\
HE & $1(3.6)$ & $4(4.3)$ & $1.20(0.13$ to 11.20$)$ & 0.87 \\
Prev features PHT & $8(26.7)$ & $44(44.0)$ & $2.16(0.88$ to 5.32$)$ & 0.09 \\
Albumin* & $44(42-47)$ & $39(32-44)$ & $0.83(0.75$ to 0.92$)$ & 0.01 \\
Child's Pugh & $9(9-10)$ & $10(9-12)$ & $1.26(1.19$ to 2.59$)$ & 0.01 \\
\hline
\end{tabular}

Conclusion In out-patients with biopsy-proven advanced ALD, clinical but not histological factors determine prognosis. Age, persistent alcohol intake, smoking habit and serum albumin are independent poor prognostic factors. Abstinence from alcohol and smoking cessation should be the priorities in the long-term management of ALD.

Competing interests None declared.

\section{PWE-286 DIRECT SERUM MARKERS ARE MORE ACCURATE THAN SIMPLE MARKER PANELS FOR THE DETECTION OF FIBROSIS IN NON-ALCOHOLIC FATTY LIVER DISEASE (NAFLD)}

doi:10.1136/gutjnl-2012-302514d.286

${ }^{1} \mathrm{~S}$ Tanwar, ${ }^{*} \mathrm{P}$ M Trembling, ${ }^{1} \mathrm{D}$ Thorburn, ${ }^{2} \mathrm{~N}$ Guha, ${ }^{3} \mathrm{~J}$ Parkes, ${ }^{2} \mathrm{P}$ Kaye, ${ }^{4} \mathrm{~A}$ Burt, ${ }^{2} \mathrm{~S} D$ Ryder, ${ }^{2} \mathrm{G}$ Aithal, ${ }^{4} \mathrm{C}$ Day, ${ }^{1} \mathrm{~W}$ M Rosenberg. ${ }^{1}$ Centre for Hepatology, University College London (UCL), London, UK; ${ }^{2}$ National Institute for Health Research: Biomedical Research Unit, Nottingham University Hospitals NHS Trust, Nottingham, UK; ${ }^{3}$ Public
Health Sciences and Medical Statistics, University of Southampton, Southampton, UK; ${ }^{4}$ Institute of Cellular Medicine, Newcastle University, Newcastle, UK

Introduction The identification of fibrosis in patients with NAFLD is important for ascertaining prognosis and stratifying patients for emerging therapeutic interventions. Use of both direct marker panels (liver matrix components) and indirect marker panels (simple biochemical tests) have been described for the detection of fibrosis in NAFLD. The aim of this study was to compare the performance of direct and indirect serum marker panels in the detection of fibrosis in NAFLD as compared with liver biopsy.

Methods From two centres, 177 patients were recruited and underwent percutaneous liver biopsy. Fibrosis staging was assessed using Kleiner criteria by two senior liver-histopathologists. Serum at the time of biopsy was used to calculate six indirect marker panels of fibrosis (APRI, BAAT, BARD, Cirrhosis discriminate score, NAFLD fibrosis index and Fib4). These panels were compared with the ELF Test (HA, TIMP1, PIIINP) and HA alone. Diagnostic accuracy was assessed using receiver operating characteristic curves which were compared using the method of DeLong.

Results The distribution of fibrosis stages in the cohort were as follows: F0 39.5\% ( $n=70)$, F1 19.2\% ( $n=34)$, F2 17.5\% ( $n=31)$, F3 $13.6 \%(n=24)$, F4 10.2\% $(n=18)$. While ELF and HA alone had the best performance overall, the ELF test was better than HA in its ability to discriminate minimal fibrosis $(p=0.02)$ and cirrhosis $(p=0.06)$. All indirect serum markers tested had significantly worse performance than ELF in the detection of cirrhosis $(p<0.05)$.

Conclusion In patients with NAFLD, direct serum marker panels have superior performance compared to indirect marker panels allowing superior stratification and prognostication. The performance of HA alone is enhanced by the addition of PIIINP and TIMP1.

Competing interests None declared.

\section{PWE-287 BREATH VOLATILE ANALYSIS FOR THE RECOGNITION OF HARMFUL DRINKING, CIRRHOSIS AND HEPATIC ENCEPHALOPATHY}

doi:10.1136/gutjnl-2012-302514d.287

${ }^{1} \mathrm{~T}$ Y Khalid, ${ }^{*}{ }^{2} \mathrm{~B}$ De Lacy Costello, ${ }^{2} \mathrm{R}$ Ewen, ${ }^{3} \mathrm{P}$ White, ${ }^{2} \mathrm{~S}$ Stevens, ${ }^{4} \mathrm{~F}$ Gordon, ${ }^{4} \mathrm{P}$ Collins, ${ }^{4} \mathrm{~A}$ McCune, ${ }^{5} \mathrm{~A}$ Shenoy, ${ }^{6} \mathrm{~S}$ Shetty, ${ }^{2} \mathrm{~N} M$ Ratcliffe, ${ }^{1} \mathrm{C} S$ Probert. ${ }^{1}$ Department of Gastroenterology, University of Liverpool, Liverpool, UK; ${ }^{2}$ Centre for Research in Biosciences, University of the West of England, Bristol, UK; ${ }^{3}$ Department of Mathematical Sciences, University of the West of England, Bristol, UK; ${ }^{4}$ University Hospitals Bristol NHS Trust, Bristol, UK; ${ }^{5}$ Colchester Hospital NHS Foundation Trust, Essex, UK; ${ }^{6}$ Dudley Group of Hospitals NHS Foundation Trust, Dudley, UK

Introduction Hepatic encephalopathy (HE) is a neuropsychiatric state which may complicate cirrhosis following the accumulation of toxic substances that cross the blood-brain barrier and affect brain function. Cirrhosis or portal-systemic shunting results in the accumulation of substances in the blood that may undergo alveolar gas exchange to be excreted in the breath. The aim of this work was to

Abstract PWE-286 Table 1

\begin{tabular}{|c|c|c|c|c|}
\hline Test & F0 vs F1-4 AUROC (95\% CI) & F0-1 vs F2-4 AUROC (95\% Cl) & F0-2 vs F3-4 AUROC (95\% Cl) & F0-3 vs F4 AUROC (95\% CI) \\
\hline Fib4 & 0.692 (0.614 to 0.771$)$ & $0.783(0.715$ to 0.851$)$ & $0.846(0.755$ to 0.937$)$ & $0.846(0.754$ to 0.938$)$ \\
\hline APRI & $0.708(0.631$ to 0.784$)$ & $0.728(0.652$ to 0.803$)$ & $0.814(0.707$ to 0.921$)$ & $0.813(0.706$ to 0.921$)$ \\
\hline NAFLD FS & $0.673(0.594$ to 0.753$)$ & $0.755(0.684$ to 0.826$)$ & $0.856(0.782$ to 0.931$)$ & $0.856(0.781$ to 0.930$)$ \\
\hline CDS & $0.584(0.501$ to 0.668$)$ & $0.588(0.501$ to 0.675$)$ & $0.634(0.491$ to 0.778$)$ & $0.631(0.487$ to 0.775$)$ \\
\hline BARD & $0.644(0.560$ to 0.728$)$ & $0.616(0.532$ to 0.700$)$ & $0.730(0.602$ to 0.858$)$ & $0.733(0.606$ to 0.860$)$ \\
\hline BAAT & $0.600(0.515$ to 0.684$)$ & $0.660(0.577$ to 0.743$)$ & $0.522(0.377$ to 0.668$)$ & $0.517(0.372$ to 0.663$)$ \\
\hline HA & $0.716(0.641$ to 0.791$)$ & $0.804(0.739$ to 0.868$)$ & $0.915(0.862$ to 0.968$)$ & $0.911(0.856$ to 0.965$)$ \\
\hline ELF & $0.759(0.689$ to 0.828$)$ & $0.813(0.747$ to 0.879$)$ & $0.942(0.902$ to 0.981$)$ & $0.937(0.896$ to 0.979$)$ \\
\hline
\end{tabular}


investigate the use of breath analysis as a non-invasive and simpler means of diagnosing HE, cirrhosis and harmful drinking.

Methods A bespoke breath-sampling device was used to sample one litre of breath through adsorbent tubes from patients with alcoholrelated cirrhosis with $(n=11)$ and without $H E(n=23)$, non-alcoholic cirrhosis without HE $(n=19)$, harmful drinkers without cirrhosis $(n=7)$, inflammatory respiratory disease $(n=18)$, and healthy controls $(n=15)$. Compounds trapped on these tubes were released via thermal desorption and analysed by gas chromatography mass spectrometry for separation and detection. Multivariate discriminant analysis was used to identify volatile organic compounds to differentiate patients according to disease status and build models for disease classification. Results Models based on the presence or absence of volatiles were tested in the patient groups. HE was correctly classified in $91.0 \%$ of patients with alcoholic cirrhosis. Patients with cirrhosis could be discriminated from those without cirrhosis with 100\% accuracy in drinkers. In patients without clinical signs of $\mathrm{HE}$, alcohol was correctly predicted as the underlying cause of cirrhosis in $82.6 \%$ of patients and non-alcoholic causes of cirrhosis were correctly determined in $84.2 \%$ of patients. Non-alcoholic cirrhosis, alcoholic cirrhosis, and harmful drinking could also be discriminated from healthy controls with a sensitivity of $89.5 \%, 97.1 \%$ and $100 \%$, respectively.

Conclusion Breath volatiles can be used to aid the diagnosis of HE, cirrhosis, and harmful levels of drinking, therefore breath testing may offer a means to detect liver conditions non-invasively at earlier and more treatable stages.

Competing interests None declared.

\section{PWE-288 SPONTANEOUS BACTERIAL PERITONITIS: PREVALENCE ON ADMISSION TO A TERTIARY CENTRE AND SUBSEQUENT OUTCOME}

doi:10.1136/gutjnl-2012-302514d.288

T Bugeja, * W T Gelson, W J H Griffiths. Department of Hepatology, Addenbrooke's Hospital, Cambridge, UK

Introduction Spontaneous bacterial peritonitis (SBP) is a sinister complication of cirrhosis associated with poor survival (approximately $38 \%$ at 1 year $^{1}$ ). However, a diagnosis of SBP does not represent, in its own right, an indication for liver transplantation in the UK under current listing criteria.

Methods We sought to investigate the prevalence and subsequent mortality in patients with an admission diagnosis of SBP. We retrospectively identified 366 consecutive cases admitted with ascites to our liver unit during the calendar year 2009. Of these 271 $(74 \%)$ patients underwent diagnostic paracentesis at time of admission and were included for further analysis.

Results Of the 271 cases, 26 (9.6\%) were diagnosed with SBP at admission on the basis of an ascitic fluid white cell count above 250 cells $/ \mathrm{mm}^{3}$ (25 cases). Patients with positive cultures but no white cell response were only included if symptomatic (one case). Alcohol was the underlying aetiology in 17 cases (65.4\%) and clinical presentations were as follows: routine paracentesis (14 cases), associated pleural effusion (four cases), variceal haemorrhage (three cases), abdominal pain (two cases), jaundice (two cases), encephalopathy (one case). Ascitic culture on two patients grew gramnegative bacilli, both resistant to quinolones being used for prophylaxis. One ascitic culture grew Lactobacillus spp. and one a mixed growth of Enterococcus faecalis and gram-positive cocci. Three patients $(11.5 \%)$ died during the index admission. Three patients $(11.5 \%)$ had successfully undergone liver transplantation and were alive 6 months after admission. Overall mortality at 6 months from an admission diagnosis of SBP was $50 \%$.
Conclusion SBP is not uncommon in cirrhotic patients with ascites, can often present silently and is associated with high mortality. Resistance to standard quinolone prophylaxis and isolation of grampositive bacteria are more recent phenomena in this group of patients. All cirrhotics admitted with significant ascites should undergo diagnostic paracentesis to exclude SBP and assessment for liver transplantation must be an urgent consideration in appropriate candidates. Listing criteria may need to be revised to include SBP as a standard indication.

Competing interests None declared.

\section{REFERENCE}

1. Tito $\mathbf{L}$, et al. Recurrence of spontaneous bacterial peritonitis in cirrhosis: frequency and predictive factors. Hepatology 1988;8:27-31.

\section{PWE-289 CRITICAL ILLNESS EARLY WARNING SCORES RETAIN ACCURACY IN PATIENTS WITH LIVER DISEASE-AN ANALYSIS OF 182000 INPATIENT OBSERVATION SETS}

doi:10.1136/gutjnl-2012-302514d.289

${ }^{1} \mathrm{~T}$ Hydes, ${ }^{2} \mathrm{P}$ Schmidt, ${ }^{3} \mathrm{G}$ B Smith, ${ }^{4} \mathrm{D}$ Prytherch, ${ }^{1} \mathrm{R} \mathrm{J}$ Aspinall. ${ }^{1}$ Department of Gastroenterology and Hepatology, Queen Alexandra Hospital, Portsmouth, UK; ${ }^{2}$ Acute Medicine, Queen Alexandra Hospital, Portsmouth, UK; ${ }^{3}$ School of Health \& Social Care, Bournemouth University, Bournemouth, UK; ${ }^{4}$ University of Portsmouth Centre for Healthcare Modelling and Informatics, Queen Alexandra Hospital, Portsmouth, UK

Introduction Aggregate weighted track and trigger systems (AWTTS) can identify critically ill patients at high risk of mortality. However, these scores use features of the Systemic Inflammatory Response Syndrome (SIRS) that may be altered in liver disease such as resting tachycardia and hypotension secondary to hyperdynamic circulation, blunted pyrexial response or hyperventilation due to encephalopathy. These scores have not been evaluated in patients with liver disorders. We therefore examined whether a range of AWTTS, including the VitalPac Early Warning Score (ViEWS), retain accuracy in liver disease.

Methods Clinical observations were recorded on a computerised database (VitalPac) for all admissions between July 2006 and April 2011 in a large hospital serving a population of 650000 . Adults assigned International Classification of Diseases (ICD-10) codes for liver disease, either as primary or comorbid diagnosis, were identified. ViEWS scores of $0-19^{1}$ were allocated to all vital sign sets. Each set contained: date/time, pulse, blood pressure, respiration, temperature, neurological status using either the Alert-Verbal-Painful-Unresponsive scale or Glasgow Coma Score, pulse oximetry and use of supplemental oxygen. Datasets were analysed with respect to number of patients needed to be seen by a doctor if escalation occurred at that score and inpatient mortality within $24 \mathrm{~h}$.

Results We identified 44328 observation sets for patients with a primary liver diagnosis (PLD) code of which 519 (1.17\%) were followed by death within $24 \mathrm{~h}$. For those with a non-primary liver diagnosis (NPLD) 138217 observations were made and 1157 (0.84\%) resulted in death. There were no differences at any ViEWS score in the prediction of 24-h mortality and number needed to be seen for patients assigned a PLD or NPLD, compared to all adult hospital admissions (Abstract PWE-289 figure 1). The area under the receiveroperating characteristics curve (95\% CI) was 0.886 for all patients and 0.888 and 0.883 for those with PLD and NPLD respectively.

Conclusion Using an electronic database of all clinical observations and diagnostic codes, we found the accuracy of predicting death within $24 \mathrm{~h}$ was retained in the presence of liver disease regardless of primary or secondary diagnosis. We have now expanded this work to include analysis of 34 additional AWTTS scores and distinct clinical presentations of hepatic disorders.

Competing interests T Hydes: None declared, P Schmidt Shareholder with: Director of a company with a minority shareholding in the development of Learning Clinic 